\title{
A Proposal to Apply Simple Photo-Reflector Elements to the Identification of Cans
}

\author{
Member Ryuji Igarashi (Akita University) \\ Member Makoto Nishida (Akita University)
}

Keywords : Photo-Reflector, Painted Color, Identification, Color Surface, Similarity

\section{Introduction}

A proposal for identifying cans is given of the use of simple photo-reflector(PR) elements. Sample cans practiced are those which are distributed to vending machines.

\section{Setup and Procedure}

The central part of the identification system is a PR element(p5589:Hamamatsu) consisting of an LED and a phototransistor(P-TRS). As represented schematically in Fig.-1(a), light emitted from the LED is reflected on the surface of the sample can, and then arrives at the P-TRS. The amount of the light which reaches the test surface depends upon the geometrical factor $G_{\text {LED }}$ between the LED and the surface. Similarly the final amount of the light which activates the P-TRS is also dependent upon the geometrical factor $G_{\text {PTRS }}$ between the surface and the P-TRS. Let the emission spectra of the LED be $E_{\text {LED }}(\lambda)$, the reflection spectra on the surface be $R_{\text {surf }}(\lambda)$, and the spectral sensitivity of the P-TRS be $S_{\text {PTRS }}(\lambda)$. Then the P-TRS current $I_{\mathrm{p}}(\lambda)$ is expressed as

$$
I \mathrm{P}(\lambda)=G_{\mathrm{LED}} E_{\mathrm{LED}}(\lambda) R_{\text {surf }}(\lambda) G_{\mathrm{PTRS}} S_{\mathrm{PTRS}}(\lambda)
$$

When the combined sensitive-range of the light concerned is between $\lambda_{1}$ and $\lambda_{2}$, and the conversion coefficient of the $I \rightarrow V$ converter is $A_{\mathrm{IV}}$, then the voltage output $v_{0}$ becomes

$$
\begin{aligned}
v_{0} & =A \operatorname{rv} \int_{\lambda_{1}}^{\lambda_{2}} \operatorname{IP}(\lambda) \mathrm{d} \lambda \\
& =A \mathrm{vV}\left(G_{\mathrm{LED}} G_{\mathrm{PTRS}}\right) \int_{\lambda_{1}}^{\lambda_{2}} E_{\mathrm{IED}}(\lambda) R_{\mathrm{surf}}(\lambda) S_{\mathrm{PTRS}}(\lambda) \mathrm{d} \lambda
\end{aligned}
$$

In the setup the product $A_{\mathrm{IV}} G_{\mathrm{IED}} G_{\mathrm{PTRS}}$ is regarded as a constant $K$, and the function shapes of both $E_{\text {LED }}(\lambda)$ and $S_{\text {PTRS }}(\lambda)$ are kept unchanged. The only quantity which changes in eq.(2) is $R_{\text {surf }}(\lambda)$ because the reflected spot and, therefore, the reflection property changes when the can is replaced or the (a)

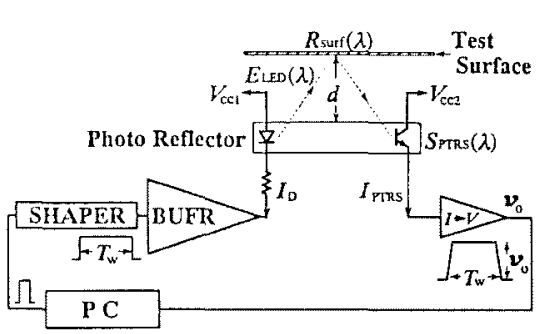

(b)

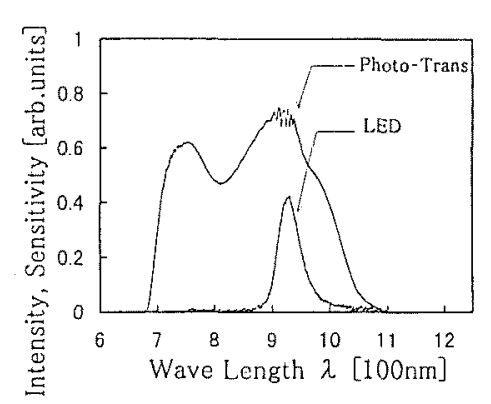

(c)

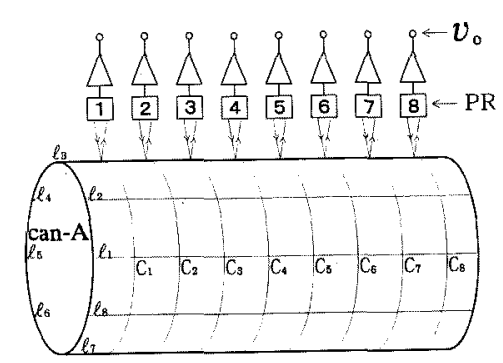

Fig.1 The illustration of the setup, spectral property, and the measuring procedure.

spot is shifted. From this consideration eq.(2) is represented as

$$
v_{0}=K v[R \operatorname{surf}(\lambda)]
$$

where

$$
v\left[R_{\text {surf }}(\lambda)\right]=\int_{\lambda_{1}}^{\lambda_{2}} E_{\mathrm{LED}}(\lambda) R_{\mathrm{surf}}(\lambda) S_{\mathrm{PTRS}}(\lambda) \mathrm{d} \lambda
$$

is a functional of the variable function $R_{\text {surf }}(\lambda)$ which represents the reflecting property of the can 
surface depending on the painted color and the coated material $^{(1)}$.

Spectral characteristics of the PR used is represented in Fig.1-(b). Although the spectral range of the LED in the PR is outside the one used in the colorimetry ${ }^{(2)}, v_{0}$ introduced in eq.(3) revealed differences in the experiments depending on the spots and cans.

Test data to identify cans were measured as explained in Fig.1-(c). Irradiation spots were selected along the circumferences $C_{1}$ through $C_{8}$. To cover eight circumferences, eight PRs were arranged along a line $l$. In the practice eight lines $l_{1}$ through $l_{8}$ were set. Slight discrepancies in $v_{0}$ between PRs were properly corrected.

\section{Results and Discussion}

In the prototype setup five different cans, can-A through can-E, were prepared for the investigation. To identify five cans, such similarity measure using Euclidean distances as explained schematically in Fig.2 was employed. In actual identification procedure, normalized distances introduced in Fig.2 were used.

$$
\begin{aligned}
& \text { Arranging } \boldsymbol{v}_{\mathrm{O}} \text { data, for example, concernig Can-A as below } \\
& \text { Along } C_{1}, \cdots, C_{\mathrm{i}}, \cdots, C_{8} \\
& \text { On } \quad l_{1} \rightarrow\left(A_{11}, \cdots, A_{\mathrm{i} 1}, \cdots, A_{81}\right) \\
& l_{\mathrm{j}} \rightarrow\left(\begin{array}{c}
\vdots \\
A_{1 \mathrm{j}}, \cdots, A_{\mathrm{ij}}, \cdots, A_{8 \mathrm{j}}
\end{array}\right) \\
& l_{8} \rightarrow\left(A_{18}, \cdots, A_{18}, \cdots, A_{88}\right) \\
& \text { A mean vector } \downarrow_{\sum A_{1 j}} \downarrow_{\sum A_{\mathrm{ij}}}^{\downarrow} \\
& a_{\mathrm{A}}=\left(\frac{\sum_{1} A_{1 \mathrm{j}}}{8}, \cdots, \frac{\sum_{1} A_{\mathrm{ij}}}{8}, \cdots, \frac{\sum_{\mathrm{j}} A_{8 \mathrm{j}}}{8}\right) \\
& =\left(A_{1}, \cdots, A_{\mathrm{i}}, \cdots, A_{8}\right) \leftarrow \text { Can-A } \\
& \text { Likely } a_{\mathrm{B}}=\left(B_{1}, \cdots, B_{\mathrm{i}}, \cdots, B_{8}\right) \leftarrow \text { Can-B } \\
& a_{\mathrm{C}}=\left(C_{1}, \cdots, C_{\mathrm{i}}, \cdots, C_{8}\right)-\mathrm{Can}-\mathrm{C} \\
& a_{\mathrm{D}}=\left(D_{1}, \cdots, D_{\mathrm{i}}, \cdots, D_{8}\right)+\text { Can-D } \\
& a_{\mathrm{E}}=\left(E_{1}, \cdots, E_{\mathrm{i}}, \cdots, E_{8}\right) \leftarrow \text { Can }-\mathrm{E} \\
& \text { Making a } \\
& \text { reference vector as } \\
& a_{\mathrm{ref}}=\left(r e f_{1}, \cdots, r e f_{\mathrm{i}}, \cdots, r e f_{8}\right) \\
& r e f_{\mathrm{i}}=\frac{A_{\mathrm{i}}+B_{\mathrm{i}}+C_{\mathrm{i}}+D_{\mathrm{i}}+E_{\mathrm{i}}}{5}
\end{aligned}
$$

Euclidean distances are derived like

$$
\begin{array}{ll}
\text { By using } & d_{\mathrm{A}}=\left|a_{\mathrm{A}}-a_{\mathrm{ref}}\right|, \ldots, d_{\mathrm{E}}=\left|a_{\mathrm{E}}-a_{\mathrm{rcf}}\right| \\
d_{\mathrm{m}}=\max \left\{d_{\mathrm{A}}, d_{\mathrm{B}}, d_{\mathrm{C}}, d_{\mathrm{D}}, d_{\mathrm{E}}\right\}
\end{array}
$$

Normalized distances are set as

$$
d_{\mathrm{AN}}=d_{\mathrm{A}} / d_{\mathrm{m}}, \ldots, d_{\mathrm{EN}}=d_{\mathrm{E}} / d_{\mathrm{m}}
$$

Fig.2 Data processing to obtain the similarity.

The result of the identification attempt is represented in Fig.3. In this instance the normalized distances differ from each other and the discrepancies well surpass the data fluctuation. Therefore, the proposed prototype system seems valid to identify cans, though further discussion is necessary to apply the system to more kinds combination of sample cans.

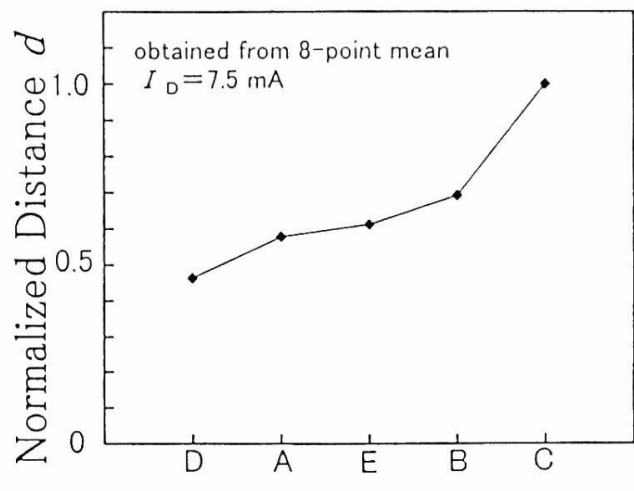

\section{Can Identification}

Fig.3 An example of identification results with the prototype system.

The present work is indebted to Miss M. Yokoi, Mr. T. Kamata, Mr. Y, Rikimaru, Mr. D. Kaminaga, Dr. K. Yamaguchi, Mr. Y. Takahashi, Mrs. M. Sugawara, Mr. Y. Iwaya and Dr. M. Sakata. The authors also appreciate the full support from Michinoku Information Co. Ltd. for carrying out the can recognition experiment.

\section{(Manuscript received June 18, 1998)}

\section{References}

(1) T. Aida : "Glossiness of Colored Papers and Its Application to Specular Glossiness Measuring Instruments", Sys. Comput. Jpn. 28, 95 (1979)

(2) G. Wyszecki and W.S. Stiles : Color Science; Concepts and Methods, Quantitative Data and Formulae, 2nd Ed. (1982) John Wiley \& Sons, New York

Ryuji igarashi (member) received the B. Eng. from Akita

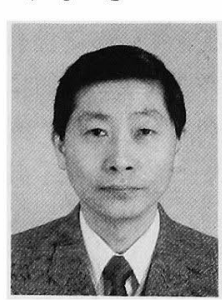
University in 1974 and was awarded the D. Eng. degree from Hokkaido University in 1986. He is an associate professor in the Department of Electric and Electronic Engineering, Akita University. $\mathrm{He}$ is a member of IEEE, the IECE of Japan, the Japan Society of Applied Physics, SICE, and Japan Society for Fuzzy Theory and Systems.

Makoto Nishida (member) received the B. Eng. from Akita

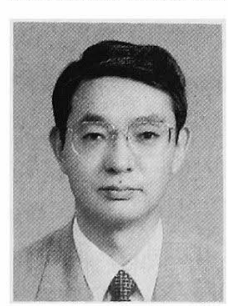
University in 1974 and was awarded the D. Eng. degree in 1984 from Tokyo Institute of Technology. Currently he is a professor in the Department of Information Engineering of Akita University. He is a member of IEEE, the Japan Society of Applied Physics, and Japan Society for Fuzzy Theory and Systems. 